\title{
Simulating Social Networks of Online Communities: Simulation as a Method for Sociability Design
}

\author{
Chee Siang $\mathrm{Ang}^{1}$ and Panayiotis Zaphiris ${ }^{2}$ \\ ${ }^{1}$ Centre for HCI Design, City University London \\ jimmybbq@gmail . com \\ ${ }^{2}$ Department of Multimedia and Graphics Art, Cyprus University of Technology
}

\begin{abstract}
We propose the use of social simulations to study and support the design of online communities. In this paper, we developed an Agent-Based Model (ABM) to simulate and study the formation of social networks in a Massively Multiplayer Online Role Playing Game (MMORPG) guild community. We first analyzed the activities and the social network (who-interacts-with-whom) of an existing guild community to identify its interaction patterns and characteristics. Then, based on the empirical results, we derived and formalized the interaction rules, which were implemented in our simulation. Using the simulation, we reproduced the observed social network of the guild community as a means of validation. The simulation was then used to examine how various parameters of the community (e.g. the level of activity, the number of neighbors of each agent, etc) could potentially influence the characteristic of the social networks.
\end{abstract}

Keywords: Agent-based model, online community, simulation, sociability, social network.

\section{Introduction}

Developing online communities involves usability design, which supports consistent, controllable and predictable Human-Computer Interaction (HCI), as well as sociability design, which focuses on human-human interaction (i.e. social interaction) and social policies [24]. The majority of research on sociability design has so far been revolved around conventional HCI methods such as query-based techniques (e.g. questionnaires, interviews), observation and content analysis to identify sociability requirements of online communities. For instance, using these methods, several studies have already analyzed the content of messages that people post in online communities [26, 13, 11, 4, 27, 25, 14, 22].

When developing online communities, we believe that it is useful to be able to evaluate and compare design alternatives. For instance, how different social policies could potentially lead to the expansion of the community; how specific interface design elements could sustain the growth of the online social networks. We might also want to find out how certain changes in an existing community could affect its future development.

One possible way of sociability design evaluation and comparison is through simulations. Suppose we have an online game community and we notice that there are a lot 
of off-topic discussions going on. We want to introduce a new policy/new design that could reduce these discussions but we are unsure whether this will be beneficial or detrimental to the community. Often it is impractical to run large-scale experiments on such a massive online community. Therefore, we are proposing a way of testing such situations through the use of simulations. In this case, we could develop a social simulation that mimics the interaction behavior of the existing community and conduct "virtual" experiment using the simulation.

Our aim was to develop an Agent Based Model (ABM) to simulate and study social networks of online communities and investigate the usefulness of simulations in sociability design. As a case study, we chose to analyze a Massively Multiplayer Online Role Playing Game (MMORPG) guild community (a virtual association with formalized membership and rank assignments that encourage participation). To achieve this, we:

- observed and analyzed the social interaction in an existing guild community

- developed an ABM which was founded on this empirical observation to simulate the community

- conducted "virtual experiments" with the simulation to demonstrate its usefulness in sociability design.

\section{Agent Based Simulation}

In term of social simulations [10], an Agent Based Model (ABM) [33] is a computational model for studying the process of the social system as a whole through simulating the actions and interactions of autonomous individuals, known as agents. Agents can have data-gathering and decision making abilities, sometimes equipped with sophisticated learning capabilities and they can be adaptive to the environment. Traditionally, sociologists have understood social life as a system of institutions and norms that shape individual behavior from top down. When dealing with linear systems, the behavior of the whole system corresponds exactly with the sum of its constituting parts (such as multiple regression models). In non-linear complex systems (such as online communities), even if the observer has a good understanding of how each part works, it will not be possible to understand the system as a whole [28]. Instead of starting from the system as a whole and decompose it (top-down), it will be more fruitful to start from its constitutive parts (bottom-up). In such systems, coherent system behaviors not defined a priori may spontaneously emerge from the aggregate dynamics of its components (in our case agents).

ABM simulation can work like a virtual laboratory through which the researcher can test hypotheses, manipulating variables and constraints of the model and observe the outcome. By generating many simulation runs with different conditions, large data sets of online communities can be accumulated. Research using ABMs has demonstrated the ability to "grow" societies "in silica" [9]. Various social phenomena have been studied using ABM techniques, including the simulation of digital markets [18], violence and conflict modeling [8, 12], trust and cooperation [19], archaeological simulation [6], ecosystem management [2], etc. 
The main strength of ABMs lies in its focus on simulating relationships between agents. Therefore, not surprisingly, ABMs have been applied to simulate interaction between agents in social networks. In most cases, ABMs are used to study two situations in social networks. Firstly, simulations can be developed to examine agents' interaction and the aggregate interaction dynamics within specific topology of social networks. Wilhite [32] for instance examined trading interaction, i.e. search, negotiation and exchange, within four types of social network topology: global network, local disconnected network, local connected network and small-world network. Instead of exploring the process of network formation, he was interested in understanding how different types of social network configurations affect trading behaviors.

More common in the research of social network simulation is the generation of social networks that arise from agents' interactions. For example, some work has been carried out to explore the network formation based on the simulation of innovation creation and knowledge diffusion in industry [1, 11, 23]. In another study, simulations were used to replicate the structural characteristics of an online discussion forum, and evaluated how different types of expert ranking algorithms may perform in communities with different characteristics $[34,35]$. ABMs have also been used increasingly in studying network evolution $[5,20]$. The study reported in this paper focused on social network generation. The ABM described in this paper simulated the interaction behaviors of individual members of a MMORPG community to understand the dynamics of the emerging social network in that community. Through the simulation we explored how various interventions could affect community performance.

\section{Methods}

The study was carried out in four phases: modeling process, simulation development, parameter setting and validation, as well as virtual experiments.

\subsection{Modelling Process}

At this stage, we analyzed a guild community of World of Warcraft (WoW) [3], a MMORPG, to understand the interaction patterns and social networks of the community. We collected player chat interaction data from a relatively large guild (with 76 members at the time of observation). Using the in-game chat-log function, we kept a record of guild members' chat activities throughout the study. A total of 1944 guild messages were collected in 30 hours of observation.

We carried out qualitative analysis of the chat interaction patterns in the guild community in order to formulate the interaction rules of the agents in the simulation. We categorized the messages using thematic and content analysis [16], focusing on three interaction types: "help provision", "help seeking" and "friendly chat". Then, we tabulated the messages from the empirical data into socio-matrices for social network analysis by identifying "who talked to whom" relationships from the chat log. Three social-matrices representing help seeking, help provision and friendly chat interaction, as well as a social-matrix representing the aggregate interaction of the three interaction types were generated for quantitative Social Network Analysis 
(SNA) [29]. Based on the results from this empirical study, we derived the "rules" of interaction patterns and formalized them into an ABM. Figure 1 shows the conceptual framework of the simulation. Agents interact with each other, resulting in the formation of ties and thus social networks (simulation output). The interaction is defined by five sets of interaction rules:

- initiation rules that determine whether an agent will initiate an interaction in a simulation time cycle

- interaction type decision rules that decide what types of interaction an agent will initiate

- help seeking, help provision and friendly chat rules: the behavior of the agents engaging in these three interaction types.

The simulation parameters (simulation input) are extrinsic factors that have an impact on all of the agents and therefore their interaction. In the context of sociability design, we can see parameters as external factors imposed on the community that are caused by design decisions or policy interventions. We explored the following five parameters and their influence on the formation of social networks in the community:

- Social budget: how does the maximum number of immediate friends an agent can have affect the social network of the whole community?

- Activeness factor: the level of activity; what will happen to the social network if all the agents of the community become more active in initiating an interaction?

- Cohesion factor: what if the agents tend to interact mostly with their friends in the social network and not with anyone outside their friends network?

- Help threshold: the ability of the agents to help someone who needs help

- Chat threshold: the tendency of the agents to engage in chatting interactions.

These parameters were derived from literature $[4,11,13]$ in order to explore the most prominent factors that affect online social networks. All parameters were scaled from 0 to 10 . Note that these are relative values for the purpose of comparison. We believe that understanding the influence of these parameters on the social network will answer practical design questions about online communities. We explain later how we used practical scenarios to explore this further.

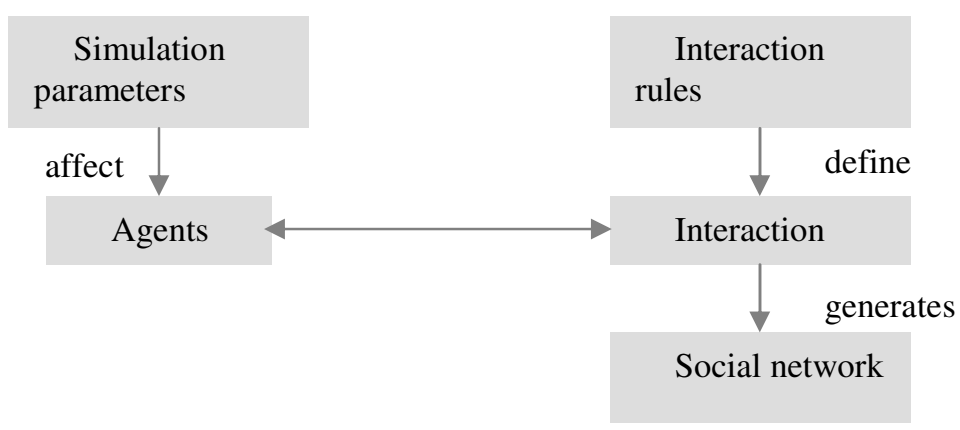

Fig. 1. The conceptual framework for the simulation 


\subsection{Simulation Development}

Figure 2 shows a snapshot of our simulation developed using Netlogo cross-platform multi-agent programmable modeling environment [31]. This snapshot shows the formation of a social network.

There are four major types of components in the interface:

- The parameters (simulation inputs): researchers can tweak the parameters of the simulation to control the process of the simulation.

- The network visualization (simulation outputs): researchers can examine the visual patterns of the social network being created; the size of the node indicates the centrality and the color brightness indicates the size of the friend network.

- Network analysis result displays (simulation outputs): includes a general network measure report, density, in- and out-degree centralization and reciprocity [29]. These results can be automatically calculated when the social network evolves or can be calculated at any point of the simulation.

- The control panel: allows researchers to start, pause and stop the simulation; to xport the social network matrices generated by the simulation for further in depth analysis with standard SNA software packages such as UCINET (http://www.analytictech.com/).

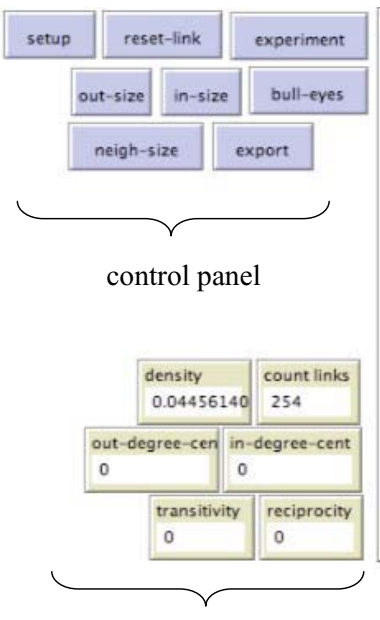

result display

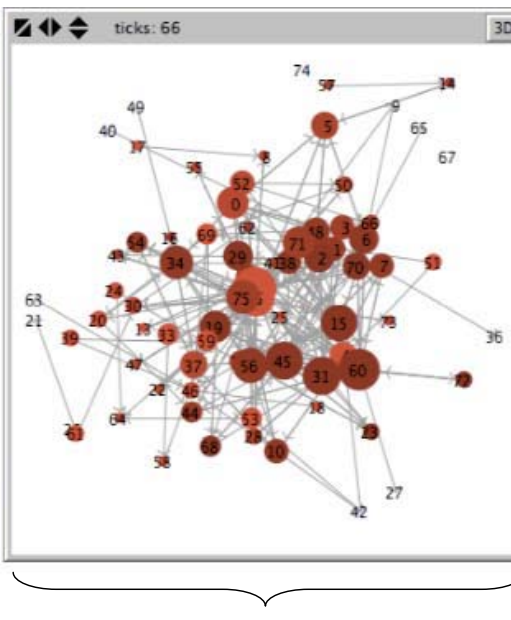

visualization

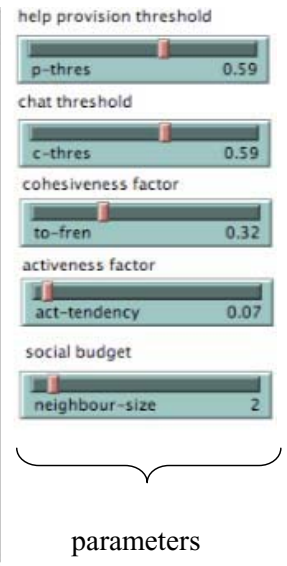

parameters

Fig. 2. The implementation of the simulation

\subsection{Parameter Setting/Calibration and Validation}

Once the simulation was programmed and verified (bugs removed) the next step was to set the initial values of the parameters of the social network to be simulated and to validate the simulation.

Much recent work on modeling of complex networks in social, biological and technological domains has focused on replicating one or more aggregate characteristics of real world networks, such as degree centrality, clustering, path lengths [21]. 
We followed this research tradition. We validated our model by producing social networks that match the aggregate characteristics of the social network of the observed WoW guild community. To achieve this, the initial values of the parameters had to be set. It was an iterative systematic process in which we compared four SNA measures (density, in- and out- degree centralization and reciprocity [29]) of simulated and observed (the aggregate network of the three interaction types as mentioned earlier) networks. We would like to emphasize that at this stage of the study, we only attempted to model this specific guild community we observed, with no intention to generalize it to other online communities.

At each iteration, we adjusted the parameters, generated 30 social networks and calculated the means of the SNA measures. Using confidence interval validation techniques, we examined the error/discrepancy between the simulated and the empirical results. We re-adjusted the parameters and repeated the same process until the error was within the tolerate level (refer table 1). This iterative process not only helped us set the initial values of the parameters, but also augmented our understanding of the parameters and the underlying structure of the model.

The validity of our simulation is demonstrated by the fact that at the final iteration of the parameter setting, the worse case error of all SNA measures is lower than the tolerate error (table 1). Furthermore, once the parameters were set and the simulation was validated to match empirical data, we demonstrated that the simulation was able to reproduce social networks observed and described in past research [30]. For

Table 1. Confident interval validation of the simulation

\begin{tabular}{|l|c|c|c|c|}
\hline & density & Out-degree & In-degree & Reciprocity \\
\hline Observed Mean & 0.0449 & 0.2247 & 0.1977 & 0.6230 \\
\hline Tolerance Error & 0.0050 & 0.0500 & 0.0500 & 0.0500 \\
\hline Simulated mean & 0.0448 & 0.2416 & 0.1836 & 0.6533 \\
\hline Standard Deviation & 0.0006 & 0.0351 & 0.0372 & 0.0324 \\
\hline Worst case error & 0.0004 & 0.0300 & 0.0280 & 0.0425 \\
\hline
\end{tabular}

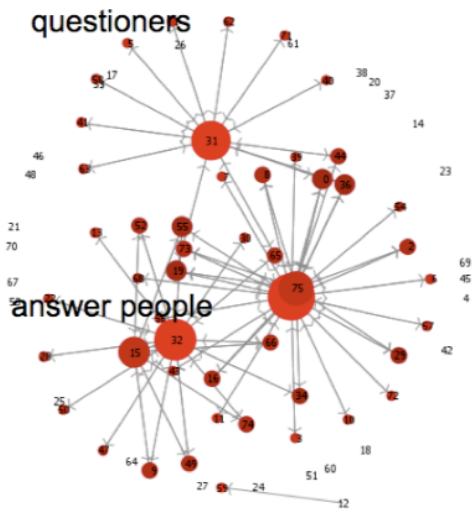

(a)

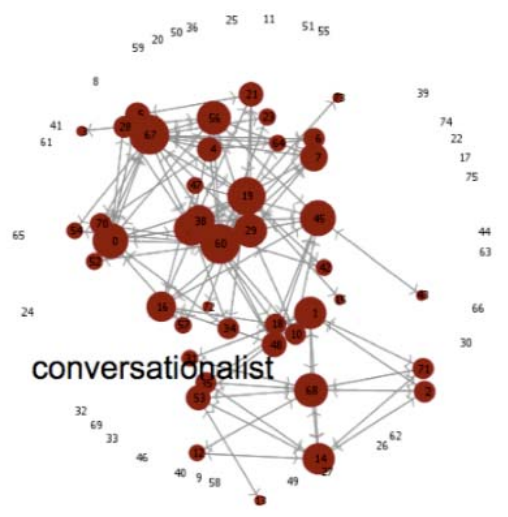

(b)

The bigger the size of the note, the higher the in-degree centrality The darker the color the bigger the local friend network

Fig. 3. (a) A typical simulation run with only "seek help" interaction activated. (b)A typical simulation run with only "friendly chat" action activated. 
instance, a star structure network emerged when we only activated "provide help" interaction rules (figure 3(a)). On the other hand, a more "cliquey" and strongly clustered network was observed when we activated only the "friendly chat" interaction rules (figure 3(b)). These results are in agreement with [30].

\subsection{Virtual Experiments}

Finally, we carried out virtual experiments with the simulation to identify the relationship between the parameters and the social network characteristics. This was done through changing the value of a specific parameter and observing the emerging social network.

The value of the five parameters was varied at 11 levels independently and 30 social networks were generated for each level. For instance, to explore the influence of the cohesion factor on the social network, we generated 30 networks for each 11 level of cohesion factor (from 1 to 10 on step 1) holding all other parameters constant. The average of the SNA measures was calculated for each set of the 30 social networks. A total of $30 \times 11 \times 5$ (parameters) = 1650 social networks were produced for the main analysis.

\section{Results and Design Implication}

In this section, we present the results of the virtual experiments and discuss how the results could be practically useful in various sociability design scenarios. Note that the results were exploratory. Instead of using the simulation to confirm hypotheses, the simulation was used to explore the dynamics of the community in relation to the parameters.

\subsection{Sociability Design Scenario 1: Friend Network}

One benefit of participating in a guild community is to make and maintain friends. We believe that the number of friends people have on an individual level might influence the structure of the whole social network. Therefore a sociability consideration of online communities is to design and facilitate friend networks. To explore this scenario, we analyzed the causality of the parameter "social budget" and the social network formation of the community. Social budget defines the maximum number of immediate friend(s) an agent could have (note that the value of parameters is relative, i.e social budget of 4 does not literally means 4 friends). The higher the social budget, the bigger the agent's friend network could be.

Figure 4 shows that when the agents had 0 social budget (i.e. no friend at all), the network density was relatively high. That was because the simulated agents were interacting randomly with everyone in the community, resulting in the creation of more ties and thus a dense network. The density dropped quite drastically when the agents had only one social budget.

As the agents were allowed more friends (higher social budget), the density rose gradually since the increase of social budget resulted in the expansion of their friend network size. However, even at social budge of 10 , the density never reached a level as high as when we had random interaction (when social budget $=0$ ). This was 


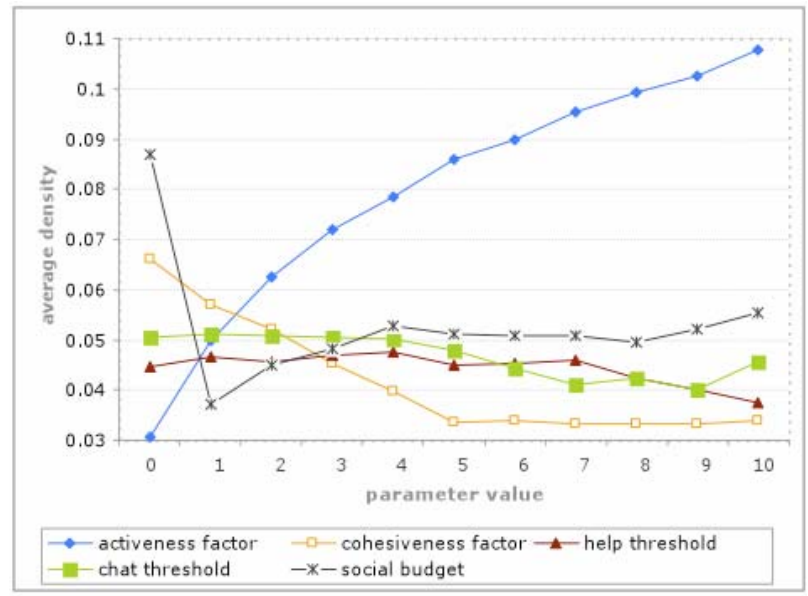

Fig. 4. The affect of the parameters on the average density of the social network

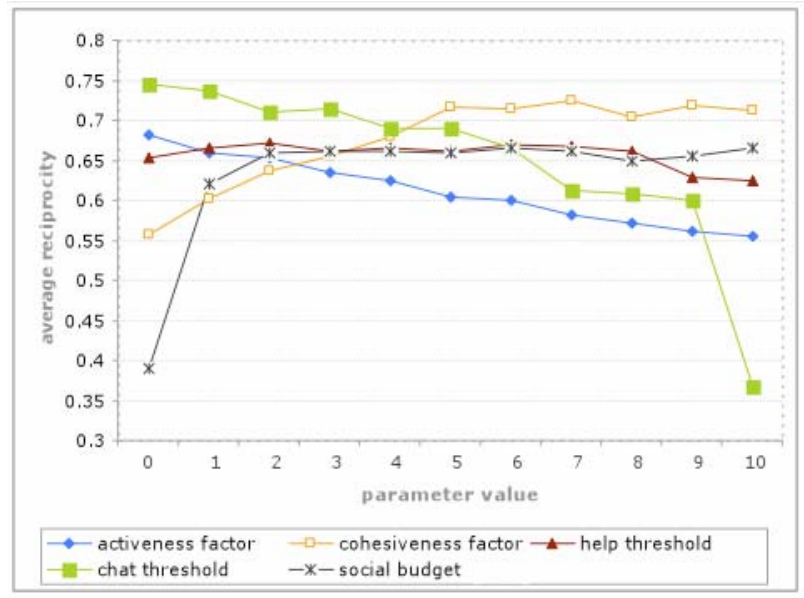

Fig. 5. The affect of the parameters on the average reciprocity

confirmed by 30 additional simulation runs from which we found that when social budget was 8 , the average size of friend networks was only 5.416.

Hence, we can safely claim that once an agent had built up a friend network, the agent would be rarely interacting with strangers (non-friends). Therefore, the friend network stopped growing. This explains why the density remained stable even when we kept increasing the social budget.

Contrary to the density, the reciprocity of the network saw a jump as social budget was increased from 0 to 1 (Figure 5). We believe that this was due to the formation of strong ties within the agent's friend network. On the other hand, when the agent was interacting randomly only with strangers (social budget $=0$ ), the chance of building reciprocated ties was low. 
Therefore, we can infer from Figure 4 and 5 that although more ties were formed when social budget was 0 , these ties were mainly unreciprocated and thus weaker ties. Once a friend network was formed, the agents interacted more frequently with their friends, resulting in more reciprocated ties. However, increasing the social budget further did not increase the reciprocity of the whole network because as previously shown, the friend networks stopped expanding.

From this analysis of the relationship between social budget and social networks, we can conclude that there is a need of a design mechanism that facilitates the formation of friend networks that promote strong ties. Moreover, the results suggest that once the agents formed a small friend network they stopped interacting with new people. Although this resulted in stronger ties, the density was compensated. Therefore, we could introduce a design that could support not only the formation but also the expansion of friend networks. For instance, a friend suggestion functions could be implemented to recommend other players who might be doing similar quests and have complementary skills.

\subsection{Sociability Design Scenario 2: Level of Activity}

One common belief is that active participation ensures the success of an online community. Therefore, one major sociability design issue is to design to motivate users to act. We explored this scenario with the parameter "activeness factor" that defines the level of activity of the community overall. The higher the activeness factor, the more likely the agents will initiate an interaction.

From all the graphs (figure 4-7), we can see that the activeness factor had a profound impact on all aspects of the social network. Firstly, we observed a continuous increase in the network density as the activeness factor was increased (Figure 4). This result was expected, since when more agents initiated an interaction, more ties were created in the network.

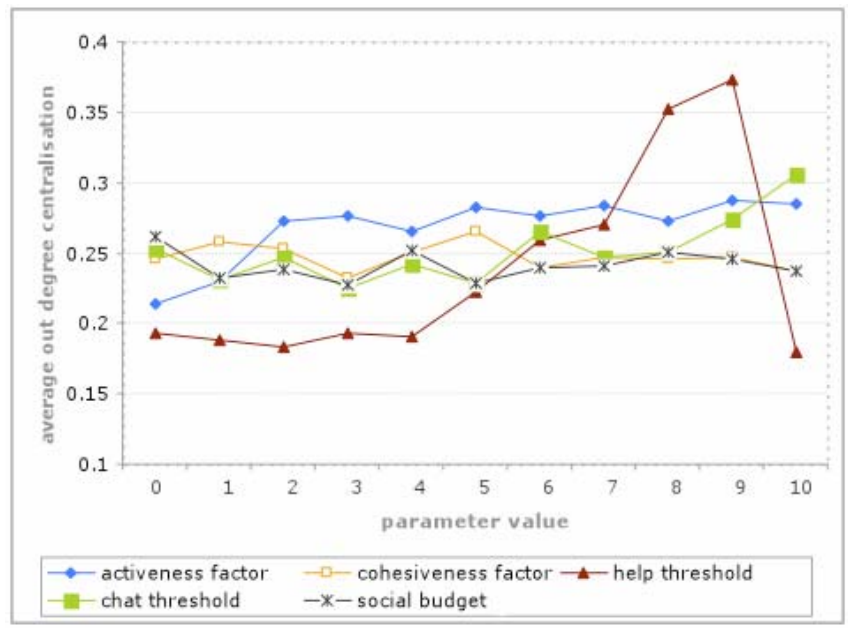

Fig. 6. The affect of the parameters on the average out-degree centralization 


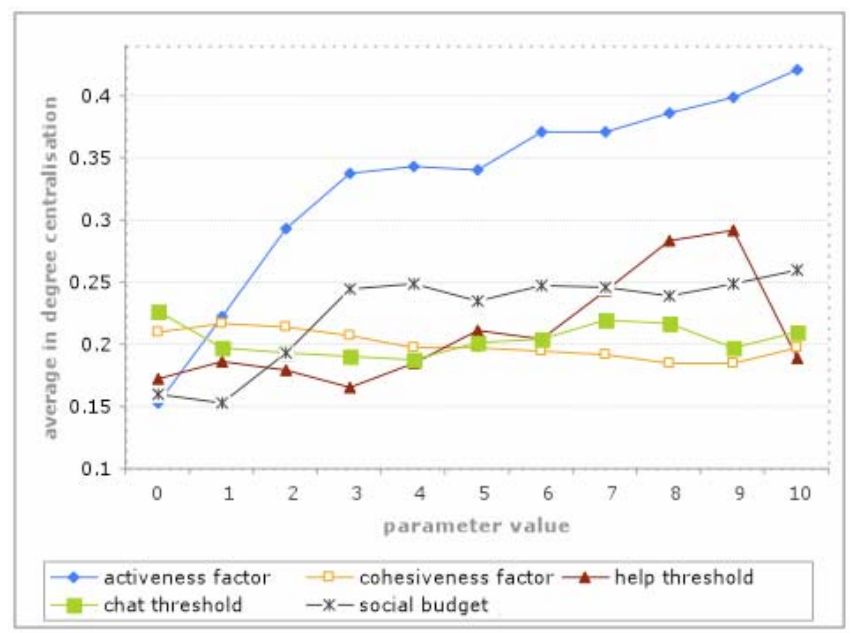

Fig. 7. The affect of the parameters on the average in-degree centralization

From Figure 6 and 7, we noticed that in-degree centralization rose much more than out-degree centralization as the activeness factor was increased. Out-degree centralization seemed to level off at 2 while in-degree centralization continued to rise.

Degree centralization can be used as a measure of equality of the network. The higher the degree centralization, the less equal the network is. This is caused by the emergence of a small "elite" group who are either popular or who dominate the interaction. The network could be unequal in term of the number of messages sent out (outdegree) and the number messages received (in-degree). Out-degree centralization can therefore be related to domination, while in-degree centralization implies popularity.

Since density increased as activeness factor went up, it is reasonable to observe a rise as well in in-degree centralization. The increase in network density (thus the number of ties formed) increased the number of messages being sent to these popular agents, amplifying the inequality in the social network.

The affect of activeness factor on out-degree centralization was rather counterintuitive because one would have expected a decrease in out-degree centralization because higher activeness factor motivated more agents to initiate an interaction. In other words, more agents would have a chance to initiate an interaction, thus lowering inequality in message sending. However the results showed an increase in out-degree centralization, suggesting otherwise.

To understand this, we examined the increase in the number of messages sent by each agent. We ran additional 60 simulations to collect data at individual level. We found that by increasing the activeness factor from 0 to 2 , the number of messages sent by each agent increased at different individual rates. Correlation analysis showed a positive relationship between the number of messages sent when activeness factor $=$ 0 and the increase in messages sent when activeness factor increased from 0 to $2, \mathrm{r}=$ $0.330, \mathrm{p}<0.05$. It means that active agents who already dominated the community sent out even more messages when the activeness factor was increased. The increase impacted inactive agents to a less extent. This created a more unequal situation in the social network, widening the activity gap between the active and inactive agents. 
As for reciprocity, we observed a gradual drop as the activeness factor increased. This was due to the fact that the increase in density was caused by an increase in the number of out-going ties instead of in-coming ties, i.e. as the agents were likely to initiate more interaction while the likelihood of reciprocating a tie stayed relatively constant.

What do these results tell us about sociability design? We found that higher activeness factor caused the formation of more ties in the community. This finding conforms to the idea that one should attempt to encourage the community members to become more active in participation. This is often achieved through the introducing ranking system that rewards all active behaviors. However, as shown in our analysis, high levels of activity could result in inequality in the community. Higher in-degree centralization suggests that only a small group of guild members benefited (through receiving more messages) from the high level of activity in the community. Depending on the types of community we are designing, this might not be desirable because inequality can often lead to social isolation and exclusion. Moreover, high level of activity also resulted in formation of weaker ties (low reciprocity). When designing a social support or empathy community, we might prefer a social network with reciprocated but fewer ties over a network with a lot of unreciprocated ties. In a collaborative writing community (such as Wiki) however we might prefer a social network with a lot of ties even if they are not reciprocated.

\section{Discussion and Conclusion}

In this paper, we proposed a new method for sociability design and research through the use of simulation. Due to space limitation, we were not able to discuss in detail the impact of all the parameters to social network formation but we hope we have demonstrated the strength of $\mathrm{ABM}$ in studying online communities. Our simulation was empirically based in two ways. Firstly, the interaction rules were formalized and drawn from the analysis of the user interaction of a WoW guild community. Secondly the simulation was validated through SNA of the interaction network of the existing community. We would also like to reiterate that the main aim of this study was to demonstrate the strengths/weakness of social simulations in sociability design and the results were largely exploratory.

As aforementioned, since we are unable to directly modify the community or its users, we need an alternative way to study how the underlying characteristic of the community influence the social network that arises. We believe that simulations allow us to understand this causal relationship by generating data which may not be possible to obtain empirically. This understanding can also enable us to design better ways to transform and expand these communities with minimal risks. Another huge advantage of $\mathrm{ABM}$ is that it is easy to conduct simulation runs and generate a large number of data. Through simulations, we can relate the simulation parameters to design decisions or social policy reinforcements.

Therefore, one obvious question is of course "how do we practically relate design/policy decisions to the change in local level interaction (the simulation parameters)", for instance "how do we know that introducing a specific reward system will lead to the increase/decrease of the activeness of the community?" We believe this 
can be done through conventional HCI methods. Existing HCI methods that focus on empirical studies/user testing can be easily integrated into simulations. Experiments can be carried out to examine the impact of a policy change or modification of graphical user interface elements on interaction behaviors at individual/micro level. For instance, we could conduct small-scale experiments with 30 people and estimate to what degree a particular "reward system" (a design decision) increases or decreases their initiative to send a message (a simulation parameter). We can then incorporate this empirical result into the simulation to predict the social network that might arise through the introduction of this reward system.

Apart from the potential strength in practical sociability design of online communities, simulations could also allow us to examine fundamental research question regarding user interaction behavior and social networks. Through the process of modeling and simulation development, we gain a deeper understanding of the mechanism of the community because the interaction rules need to be formulated very precisely to be programmable into ABMs. Therefore a possible use of simulation in sociability research is to construct theory-driven simulations. Using simulations, we can formalize social theories into precise program codes and examine the theories in the context of online communication.

The simulation presented in this paper focused on how social interactions affect network generation. A reverse way of social network simulation is to impose specific network structures and observe how these affect social interactions. For instance, we could examine the difference between a "star" and "clique" structure network in term of information diffusion in an online community. Finally, more future work needs to be done to enhance the validity of the simulation in order to use the simulation as a valid tool for sociability design. Therefore, more data needs to be collected and analyzed (e.g. include more communities, analyze the virtual actions of the players instead of just the chat log, etc)

As a conclusion, simulations are a powerful technique for understanding sociability in online communities. Our simulation was not only capable of generating results which were in accordance with what was observed in the real community, but also allowed us to explore various what-if questions that helped us to understand the sociability design tradeoffs of online communities.

\section{References}

1. Ahrweiler, P., Pyka, A., Gilbert, N.: Simulating knowledge dynamics in innovation networks (SKIN). In: Leombruni, R., Richiardi, M. (eds.) Industry and labor dynamics: The agent-based computational economics approach. World Scientific Press, Singapore

2. Antona, M., Bommel, P., Bousquet, F., Page, C.L.: Interactions and organization in ecosystem management: The use of multi-agent systems to simulate incentive environmental policies. Paper presented at the 3rd Workshop on Agent-Based Simulation., Ghent, Belgium (2002)

3. Blizzard Entertainment, World of Warcraft (2004), http: / / www. worldofwarcraft. com/ (Last retrieved, 24 July 2008)

4. Burnett, G.: Information exchange in virtual communities: A typology. Information Research: An International Electronic Journal, 5(4) (2000) 
5. Carley, K., Lee, J., Krackhardt, D.: Destabilizing Networks. Connections 24(3), 31-44 (2001)

6. Doran, J., Palmer, M., Gilbert, N., Mellars, P.: The EOS project: Modelling upper paleolithic social change. In: Gilbert, G.N., Doran, J. (eds.) Simulating societies: The computer simulation of social phenomena. UCL Press, London (1994)

7. Ducheneaut, N., Moore, R.J., Nickell, E.: Designing for sociability in massively multiplayer games: An examination of the third places of SWG. In: Other Players conference (2004)

8. Epstein, J.: Modeling civil violence: An agent-based computational approach. In: Proceedings of the National Academy of Sciences of the USA, vol. 99(3) (2002)

9. Epstein, J., Axtell, R.: Growing artificial societies. MIT Press, Boston (1997)

10. Gilbert, N., Troitzsch, K.G.: Simulation for the social scientist. Open University Press (2005)

11. Golder, S.A., Donath, J.: Socaial roles in electronic communities. Paper presented at the Association of Internet Researchers (AoIR) conference Internet Research 5.0, Brighton, England (2004)

12. Jager, W., Popping, R., Sande, H., v. d.: Clustering and fighting in two-party crowds: Simulating the approach-avoidance conflict. Journal of Artificial Societies and Social Simulation 4(3) (2001)

13. Klemm, P., Hurst, M., Dearholt, S.L., Trone, S.R.: Gender differences on internet cancer support groups. Computer Nursing 17(2), 65-72 (1999)

14. Kollock, P.: The economies of online cooperation: Gifts and public goods in cyberspace. In: Smith, M.A., Kollock, P. (eds.) Communities in cyberspace, London. Routledge (1999)

15. Kolo, C., Baur, T.: Living a virtual life: Social dynamics of online gaming. The International Journal of Computer Game Research 4(1) (2004)

16. Krippendorff, K.: Content analysis: An introduction to its methodology. Sage Publications, Newbury Park (1980)

17. Lamieri, M., Ietri, D.: Innovation creation and diffusion in a social network: An agent based approach (2004), http: //ssrn. com/abstract=937255 (Last retrieved, 09 September 2008)

18. López-Sánchez, M., Noria, X., Rodríquez-Aguilar, J.A., Gilbert, N., Shuster, S.: Simulation of digital content distribution using a multi-agent simulation approach. In: Vitria, J., Radeva, P., Aguilo, I. (eds.) Recent advances in artificial intelligence research and development, pp. 341-348. IOS Press, Amsterdam (2004)

19. Macy, M.W., Sato, Y.: Trust, cooperation, and market formation in the U.S. and Japan. Paper presented at the Proceedings of the National Academy of Sciences of the United States of America (2002)

20. MaxTsvetovat, Carley, K.: Knowing the enemy: A simulation of terrorist organizations and counter-terrorism strategies. Paper presented at the CASOS Conference, Pittsburgh, PA (2002)

21. Newman, M.: The structure and function of complex networks. Siam Review 45(2), 167 $256(2003)$

22. Niedner, S., Hertel, G., Hermann, S.: Motivation in open source projects: An empirical study among linux developers (2000), http://www.i2.psychologie.uniwuerzburg.de/ao/research/linux_study.php (Last retrieved, 24 April 2008)

23. Özman, M.: Network formation and strategic firm behaviour to explore and exploit. Journal of Artificial Societies and Social Simulation 11(17) (2007) 
24. Preece, J.: Online communities: Designing usability, supporting sociability. John Wiley and Sons, Chichester (2000)

25. Raymond, E.: The cathedral and the bazaar: Musings on Linux and open source from an accidental revolutionary. O'Reilly and Associates, Sebastopol (1999)

26. Savicki, V., Lingenfelter, D., Kelley, D.: Gender language style and group composition in internet discussion groups. Journal of Computer-Mediated Communication 2(3) (1996)

27. Turner, T.C., Smith, M.A., Fisher, D., Welser, H.T.: Picturing USENET: Mapping computer-mediated collective action. Journal of Computer-Mediated Communication 10(4) (2005)

28. Waldrop, M.: Complexity: The emerging science at the edge of chaos. Simon and Schuster, New York (1992)

29. Wasserman, S., Faust, K.: Social network analysis: Methods and applications. Cambridge University Press, Cambridge (1994)

30. Welser, H.T., Gleave, E., Fisher, D., Smith, M.: Visualizing the signatures of social roles in online discussion groups. Journal of Social Structure 8 (2007)

31. Wilensky, U.: Netlogo: Centre for Connected Learning and Computer Based Modelling Northwestern University, Evanston, IL (1999)

32. Wilhite, A.: Bilateral trade and "small-world" networks 18, 49-64 (2001)

33. Wooldridge, M., Jennings, N.R.: Intelligent agents: Theory and practice. Knowledge Engineering Review 10(2), 115-152 (1995)

34. Zhang, J., Ackerman, M., Adamic, L.: Communitynetsimulator: Using simulations to study online community networks. Paper presented at the C\&T2007, Lansing, MI (2007a)

35. Zhang, J., Ackerman, M., Adamic, L.: Expertise networks in online communities: Structure and algor. Paper presented at the WWW 2007, Canada (2007b) 